\title{
A new approach to safe infant sleep
}

The unexpected death of an infant during sleep is still a challenge to our knowledge and practice. The international scientific community has advanced a lot in the knowledge of its mechanisms and the risk factors that should be avoided. However, after the major reduction in mortality rates following the first prevention campaigns in the 1990s, no significant changes have been observed since approximately 2000 . There has only been a mild reduction in the number of cases diagnosed with sudden unexpected infant death, together with a slight increase in the number of suffocation or strangulation diagnoses. ${ }^{1}$

Although it could be said that records are deficient, that diagnoses are inaccurate, that the setting of death is not thoroughly studied, and that autopsies are uncommon, such shortcomings have existed for many years, with subtle distinctions, and affect the accuracy of before and after values.

The triple-risk model, although not perfect like any simplified concept, allows for a schematic approach to the problem and its current situation.

\section{What do we know so far?}

1. A vulnerable child exposed to an environmental risk factor during their first year of life may die during sleep due to a failure in the microarousal mechanism in the case of asphyxia.

2. A vulnerable child is defined as a child with poor intrauterine growth and development, with immature neurological connections in relation to the response to asphyxia. This is the case of children with chronic intrauterine hypoxia due to hypertension, placental insufficiency, exposure to tobacco, alcohol or illegal drugs, stress or a poorly controlled pregnancy, and also children with genetic causes involving neurotransmission mechanisms.

3. During the first year of life, neurodevelopment and growth occur very fast, making the importance of breastfeeding, health checkups, and a complete immunization schedule noteworthy.

4. Risk factors include a prone sleeping position, the presence of loose or soft objects next to the child, overheating, and exposure to tobacco, alcohol, and illegal drugs.
5. Some recommendations to reduce such risks have been considered controversial, such as pacifier use, co-sleeping, sleep positioners or devices for their use in the parents' bed. ${ }^{2}$

\section{From theory to practice}

The first question is why, although we are aware of this information, we have not been able to reduce the mortality rate in recent years.

More controls are required during pregnancy so that children are born with fewer vulnerabilities. However, in reality, an increase has been observed in teenage pregnancy, drug, tobacco and alcohol use among young women, the number of people in unfavorable socioeconomic conditions, medically-assisted fertilization procedures for women with problems to conceive and maintain a pregnancy, and many other current realities that affect the different stages of pregnancy.

Any effort made in favor of children's growth and development during their first year of life is not only beneficial in relation to safe sleep but also provides an opportunity for a healthy life, for example, promoting and protecting breastfeeding, facilitating access to a complete immunization schedule for all children, ensuring well-child care visits and adequate disease control. ${ }^{3}$

Risk factors entail a problem, or rather, several problems. One is uncertainty. It is not always possible to identify a vulnerable child. A healthy, term newborn infant may be vulnerable due to genetic causes, such as neurotransmitter synthesis, release or reuptake deficiency. For this reason, the recommendations on safe sleep are the same for all children.

Another aspect is the lack of knowledge among the health care team members. At the maternity ward, there are usually only a few vulnerable children among normal newborn infants, but at the neonatal intensive care unit, practically every child is vulnerable.

Children born prematurely, with a low birth weight, with intrauterine growth restriction, genetic disorders or severe neonatal conditions are clearly vulnerable. The families of these children should receive training to provide them with a safe sleep environment, among other aspects. This is the role of the health care team. Nurses spend a lot of time with the families during hospitalization; they are the 
direct caregivers of newborn infants and become undisputed role models for parents. However, while some health care providers are unaware of safe sleep recommendations, others know them but fail to implement them. ${ }^{4}$ The same thing could be said about the families. ${ }^{5}$

At this point, we need to question ourselves about what we could do in response to these situations.

The Global Action and Prioritisation of Sudden Infant Death (GAPS) project was an international consensus process set up in 2015 to define research development priorities in order to reduce the rate of sudden unexpected deaths in infancy. ${ }^{6}$

After three discussion panels, it was concluded that the following is necessary:

1. To better understand the biological mechanisms underlying sudden unexpected infant death and how environmental factors interact with such mechanisms at different ages and stages of maturation.

2. To ensure best practice data collection, management, and sharing.

3. To better identify the most vulnerable populations and have a more effective communication of risks to the extent that social and cultural factors affect the family choice in sleep practices.

The American Academy of Pediatrics updated its recommendations for a safe sleep environment in 2016 to include new evidence on skin-to-skin care for newborn infants, bedside and in-bed sleepers, sleeping on couches/armchairs and in sitting devices, and use of soft bedding after 4 months of age.

Consistent with the new trends on how these recommendations should be conveyed, the emphasis was placed on encouraging an open and nonjudgmental conversation with families about their sleep practices. The specific situations of each family call for a customized message that weighs the relative risks and benefits. ${ }^{7}$

We are now leaving behind an outright message and entering the process of shared decision making, taking into consideration scientific evidence in a personal and family setting that encompasses social, financial, and cultural aspects. ${ }^{8}$

All these aspects influence how each family looks after their children. Rigid mandates lead to a rift. An open communication considering the particular family's beliefs and conditions will allow to suggest the best way to reduce the risk within the scope of their possibilities.

Without a doubt, this is more time-consuming but the best possible way forward.

Norma Elena Rossato, M.D.

Task force on "Sleep dizsorders and sudden unexpected infant death"

http:/ / dx.doi.org/10.5546/ aap.2019.eng.354

To cite: Rossato NE.A new approach to safe infant sleep. Arch Argent Pediatr 2019;117(6):354-355.

\section{REFERENCES}

1. Moon RY; Task Force On Sudden Infant Death Syndrome. SIDS and Other Sleep-Related Infant Deaths: Evidence Base for 2016 Updated Recommendations for a Safe Infant Sleeping Environment. Pediatrics. 2016; 138(5):e20162940.

2. Task Force On Sudden Infant Death Syndrome. SIDS and Other Sleep-Related Infant Deaths: Updated 2016 Recommendations for a Safe Infant Sleeping Environment. Pediatrics. 2016; 138(5):e20162938.

3. Hauck FR, Tanabe KO. Beyond "Back to Sleep": Ways to Further Reduce the Risk of Sudden Infant Death Syndrome. Pediatr Ann. 2017; 46(8):e284-90.

4. Newberry JA. Creating a Safe Sleep Environment for the Infant: What the Pediatric Nurse Needs to Know. J Pediatr Nurs. 2019; 44:119-22.

5. Hwang SS, Corwin MJ.Safe Infant Sleep Practices: Parental Engagement, Education, and Behavior Change. Pediatr Ann. 2017; 46(8):e291-6.

6. Hauck FR, McEntire BL, Raven LK, Bates FL, et al. Research Priorities in Sudden Unexpected Infant Death: An International Consensus. Pediatrics. 2017; 140(2):e20163514.

7. Altfeld S, Peacock N, Rowe HL, Massino J, et al. Moving Beyond "Abstinence-Only" Messaging to Reduce SleepRelated Infant Deaths. J Pediatr. 2017; 189:207-12.

8. Gray B, Coker TR. When shared decision-making and evidence based practice clash: Infant sleep practices. J Paediatr Child Health. 2019 Jul 29. doi: 10.1111/jpc.14577. [Epub ahead of print] 